\title{
BMJ Open Settings and monitoring of mechanical ventilation during physical therapy in adult critically ill patients: protocol for a scoping review
}

\author{
Felipe González-Seguel, ${ }^{\oplus 1,2}$ Agustín Camus-Molina, ${ }^{\oplus 1,2}$ \\ Anita Jasmén Sepúlveda, ${ }^{3}$ Rodrigo Pérez Araos, ${ }^{\odot 4}$ Jorge Molina Blamey, 2 \\ Jerónimo Graf Santos ${ }^{\oplus}$
}

To cite: González-Seguel F, Camus-Molina A, Jasmén Sepúlveda A, et al. Settings and monitoring of mechanical ventilation during physical therapy in adult critically ill patients: protocol for a scoping review. BMJ Open 2019;9:e030692. doi:10.1136/ bmjopen-2019-030692

- Prepublication history and additional material for this paper are available online. To view these files, please visit the journal online (http://dx.doi. org/10.1136/bmjopen-2019030692).

Received 27 March 2019 Revised 06 August 2019 Accepted 12 August 2019

Check for updates

(C) Author(s) (or their employer(s)) 2019. Re-use permitted under CC BY-NC. No commercial re-use. See rights and permissions. Published by BMJ.

For numbered affiliations see end of article.

Correspondence to

Felipe González-Seguel;

feligonzalezs@udd.cl

\section{ABSTRACT}

Introduction Early mobilisation has been extensively advocated to improve functional outcomes in critically ill patients, even though consistent evidence of its benefits has remained elusive. These conflicting results could be explained by a lack of knowledge on the optimal dosage of physical therapy and a mismatch between ventilatory support and exercise-induced patient ventilatory demand. Modern mechanical ventilators provide realtime monitoring of respiratory/metabolic variables and ventilatory setting that could be used for physical therapy dosage or ventilatory support titration, allowing individualised interventions in these patients. The aim of this review is to comprehensively map and summarise current knowledge on adjustments of respiratory support and respiratory or metabolic monitoring during physical therapy in adult critically ill mechanically ventilated patients.

Methods and analysis This is a scoping review protocol based on the methodology of the Joanna-Briggs-Institute. The search strategy will be conducted from inception to 30 June 2019 as a cut-off date in PubMed, CINAHL, Rehabilitation \& Sport Medicine, Scielo Citation Index, Epistemónikos, Clinical Trials, PEDro and Cochrane Library, performed by a biomedical librarian and two critical care physiotherapists. All types of articles will be selected, including conference abstracts, clinical practice guidelines and expert recommendations. Bibliometric variables, patient characteristics, physical therapy interventions, ventilator settings and respiratory or metabolic monitoring will be extracted. The identified literature will be analysed by four critical care physiotherapists and reviewed by a senior critical care physician.

Ethics and dissemination Ethical approval is not required. The knowledge-translation of the results will be carried out based on the End-of-Grant strategies: diffusion, dissemination and application. The results will be published in a peer-review journal, presentations will be disseminated in relevant congresses, and recommendations based on the results will be developed through training for mechanical ventilation and physical therapy stakeholders.
Strengths and limitations of this study

- This is the first review that seeks to identify the greatest amount of information related to mechanical ventilation settings and monitoring during physical therapy interventions.

- This study follows a recommended method to answer research questions through a scoping review.

- A biomedical librarian will perform systematic search of the databases and two independent physiotherapists will carry out the study selection.

- The databases will be selected according to the availability of our institution. To minimise the possibility of missing relevant articles, we will carry out a hand search of the reference lists of the relevant articles and a search in a grey literature database.

- The search is limited to documents in English and Spanish.

\section{INTRODUCTION}

Mechanical ventilation (MV) is one of the most common interventions in the intensive care unit (ICU). ${ }^{1}$ Goals of MV include alleviation of the work of breathing and gas exchange enhancement while avoiding additional lung or respiratory muscle injury. ${ }^{2}$ Positive pressure ventilatory support must synchronically interact with the negative pressure generated by the respiratory muscles to achieve the objectives of $\mathrm{MV}^{3}$ There are several ventilatory strategies to improve patient-ventilator synchrony during stationary conditions (eg, assisted or proportional modes), ${ }^{4}$ but little is known about ventilatory support during physical therapy.

Physical therapy is frequently restricted in mechanically ventilated patients, ${ }^{56}$ though early mobilisation has been extensively advocated to improve functional outcomes. ${ }^{7}$ Physical therapy interventions include passive movements of the extremities for deeply 
sedated patients, ${ }^{8}$ in-bed and out-of-bed mobility, ${ }^{9}$ active or passive cycling, ${ }^{10}$ neuromuscular electrical stimulation ${ }^{11}$ and ambulation. ${ }^{12}$ Clinical trials of these physical therapy interventions have reported inconsistent effects on functional outcomes in mechanically ventilated patients. ${ }^{913-17}$ These results could be explained by a lack of knowledge on the optimal dosage of physical therapy for ICU patients (in terms of intensity, duration and frequency). ${ }^{18-21}$ Alternatively, during physical therapy a mismatch between ventilatory support and exercise-induced ventilatory demand may excessively increase work of breathing, limiting physical therapy performance and eventually leading to fatigue. ${ }^{22}$

Despite expert consensus and recommendations on active mobilisation of mechanically ventilated patients, ${ }^{23}$ ventilator settings and safety parameters to monitor during mobilisation have not yet been defined. Some authors have suggested strategies to adjust ventilatory support during physical therapy; for example, increasing support level during pressure support ventilation, ${ }^{24}$ using proportional ventilation modes, ${ }^{22}$ employing assist-control ventilation before and after physical therapy ${ }^{25}$ or increasing the fraction of inspired oxygen..$^{25}$ In addition, mechanical ventilators allow real-time monitoring of respiratory (eg, respiratory rate or minute ventilation) and metabolic variables (eg, carbon dioxide production) ${ }^{22} 26$ that could provide useful information to individualise the dosage of physical therapy or ventilatory support. There is therefore a need to summarise the existing knowledge on the use of MV as a potential tool to provide safe and efficient physical therapy for ICU patients.

A first step for professionals to improve the decision-making during physical therapy intervention is to identify the MV monitoring and setting. Scoping review is a solid method to map research areas, providing a rigorous and systematic approach to the synthesis of knowledge, particularly useful when a body of literature has not yet been thoroughly reviewed. ${ }^{27} 28$ The aim of this review is to comprehensively map and summarise current knowledge on adjustments of respiratory support and respiratory or metabolic monitoring during physical therapy in adult critically ill mechanically ventilated patients.

\section{METHODS}

This scoping review will be based on the Joanna Briggs Institute (JBI) method ${ }^{2729}$ as initially conceived by Arksey and O'Malley. ${ }^{30}$ This project was reviewed, approved and registered by the Research and Clinical Trials Unit of the Departamento Cientifico Docente of Clinica Alemana de Santiago ( ${ }^{\circ}$ 2019-752).

This scoping review will be based on the six steps recommended by the JBI: (1) identifying the research question, (2) identifying relevant studies, (3) study selection, (4) data extraction, (5) collating, summarising and reporting the results, and (6) consultation. ${ }^{30}$ To control the quality of this review, the Preferred Reporting Items for Systematic Reviews and Meta-Analyses (PRISMA) extension for Scoping Reviews Checklist will be used. ${ }^{31}$

\section{Stage 1: identifying the research questions}

The research questions of this scoping review emerged from knowledge gaps identified by the authors when trying to adjust ventilator settings in order to maintain synchrony and avoid respiratory distress during physical therapy interventions. Additionally, modern mechanical ventilators provide a wealth of respiratory and metabolic data that could be used to monitor the physiological response to physical therapy. This information, in turn, could be used to further adjust ventilator settings or the dose of physical therapy in an individualised manner. The following research questions were structured based on the Population, Concept and Context method (PCC) ${ }^{27}$ :

1. What MV settings have been reported or studied during physical therapy interventions in mechanically ventilated adult critically ill patients?

2. What respiratory and metabolic variables have been reported or studied to monitor the physiological response to physical therapy interventions in adult mechanically ventilated critically ill patients?

\section{Stage 2: identifying relevant studies}

To identify relevant studies, a biomedical librarian (AJS) and two critical care physiotherapist (AC-M and FG-S) will search the literature based on the three stages recommended by the JBI. ${ }^{27}{ }^{29}$ First, an iterative search will be conducted in PubMed (FG-S and AC-M) using keywords and MeSH terms associated to 'mechanical ventilation', 'exercise', 'intensive care unit' and 'assisted mechanical ventilation'. Second, the keywords identified in the relevant articles of the first search will be reviewed to include them in a second search in the electronic databases selected for relevance of content and accessibility (limited to the databases available in our institution). The following relevant biomedical databases will be consulted: PubMed (NCBI), CINAHL plus with full text (EBSCO), Rehabilitation \& Sport Medicine (EBSCO), Scielo Citation Index (Clarivate), Epistemónikos, Clinical Trials, Cochrane Library (free access by Ministerio de Salud de Chile) and PEDro (see eg, of the full search strategy in online supplementary file table S1). It will be filtered by language (English and Spanish) and all types articles will be considered, including research articles, conference abstracts, clinical practice guidelines and expert recommendations. In addition, the grey literature search will be conducted using Opengrey. Third, a hand search of the reference lists of the relevant articles identified in the final search will be performed. The authors of primary studies or reviews will be contacted if additional information is deemed necessary. Bibliographical management software will be used to download and manage the reference database (EndNote Clarivate Analytics, Boston, Massachusetts, USA). The results for the initial search using relevant keywords and MeSH terms developed for each database are presented in table 1 . The final 


\begin{tabular}{lr} 
Table $1 \quad$ Initial database search results & \\
\hline PubMed (NCBI) & 675 \\
\hline CINAHL plus with full text (EBSCO) & 264 \\
\hline Epistemónikos & 105 \\
Clinical Trials & 41 \\
\hline Rehabilitation \& Sport Medicine (EBSCO) & 21 \\
Scielo Citation Index (Clarivate) & 11 \\
PEDro & 13 \\
Cochrane Library (free access by Ministerio de & 3 \\
Salud de Chile) & \\
\hline
\end{tabular}

search will be made within 2 weeks when this protocol is approved and the search cut-off date will be 30 June 2019 .

\section{Stage 3: study selection}

Two researchers (FG-S and AC-M) will independently carry out the article selection in three stages: filter by title, abstract and full text according to the selection criteria. Potential disagreements about the inclusion or exclusion of articles will be discussed and, if the discrepancy is not resolved, a third one (RPA) will judge. The 'include rather than exclude' methodology will be used to select by title, abstract and full text, to avoid potential loss of relevant information. ${ }^{32}$ This stage will be reported using the PRISMA flow chart. ${ }^{33}$

The following inclusion criteria will be used, based on the components of the PCC method ${ }^{27} 29$ (figure 1): Population-adult critically ill patients on MV for at least 48 hours, defined as any ventilation through an endotracheal tube, tracheostomy tube or non-invasive ventilation ${ }^{34}$; Concept (intervention)-physical therapy interventions during ICU stay ${ }^{35}$ performed by a physiotherapy, nurse or other allied-health professional, including (but is not limited to): passive, active or active-assisted range

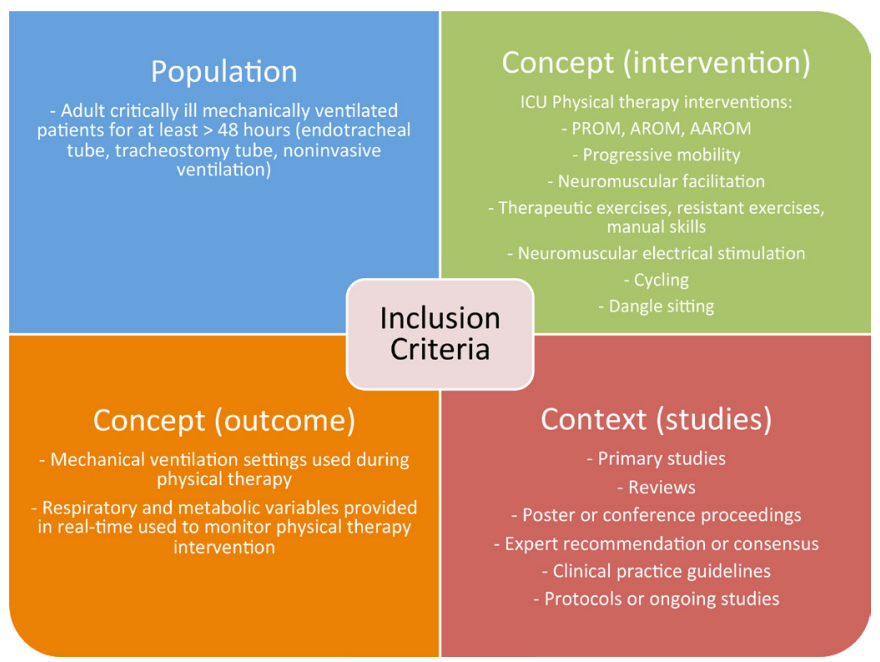

Figure 1 Inclusion criteria used in this scoping review based on the population, concept and context method. ${ }^{27}$ AROM, active range of motion; AAROM, active-assisted range of motion; ICU, intensive care unit, PROM, passive range of motion. of motion, progressive mobility, neuromuscular facilitation, therapeutic exercises, resistant exercises, manual skills, neuromuscular electrical stimulation, cycling and dangle sitting; Concept (Outcomes)-description of the MV settings used before, during and after of any physical therapy interventions. In addition, articles describing any respiratory or metabolic variables used to monitor before, during and after any physical therapy interventions will be selected; Context (studies) - all types of scientific articles, including (but not limited to) original studies, conference abstracts, reviews, clinical practice guidelines, expert recommendations and protocols or ongoing studies. The following article exclusion criteria will be used: those concerning interventions initiated outside the ICU, paediatric or neonatal population, thoracic or respiratory physiotherapy interventions (eg, inspiratory muscle threshold and airway clearance techniques), animal models, in vitro studies and articles published in languages other than English or Spanish.

\section{Stage 4: data extraction}

Relevant data extraction from the selected studies will be performed using Peters et al recommendations ${ }^{27}$ and compiled using the PCC nomenclature in a custom-made Excel 2011 (Microsoft, V.14.6.6) spreadsheet (online supplementary material table S2). Four reviewers (FG-S, AC-M, JMB and RPA) will extract the data from the qualified articles, including information from the supplementary materials and appendices. The four reviewers will complete the same data extraction form and will meet to determine if the information extracted is consistent among them to answer the questions and the purpose of the study.

The least information to be extracted from the articles will be: authors, year of publication, country, sample size, study design, objective of the study, type of publication, study population, type of ventilation used, MV settings used during physical therapy intervention (eg, ventilatory mode, mandatory respiratory rate, fraction of inspired oxygen, positive end expiratory pressure, targeted pressure or volume prescription), respiratory or metabolic variables used to monitor physical therapy (eg, respiratory rate, tidal volume, minute ventilation, oesophageal pressure or diaphragm electrical activity derived variables, pulse oximetry, breathing pattern, oxygen consumption and carbon dioxide production) and type of physical therapy intervention. Moreover, some clinically relevant causes of complications (eg, vomiting, agitation, airway trouble) will be extracted.

Physical therapy interventions will be the following ${ }^{2135}$ : passive, active or active-assisted range of motion, progressive mobility, neuromuscular facilitation, therapeutic exercises, resistant exercises, manual skills, neuromuscular electrical stimulation, cycling and dangle sitting.

Outcomes will be classified using the following definitions: MV adjustments during physical therapy session and respiratory/metabolic real-time variables used to monitor physical therapy interventions. 


\section{Stage 5: collating, summarising and reporting results}

Compiled data from the selected studies will be synthesised by four researchers (FG-S, AC-M, JMB and RPA) in a logical and descriptive order aligned with the study questions. According to the methodology described by Arksey and O'Malley, ${ }^{30}$ we anticipate that the extraction and synthesis of the data will be an iterative process that will depend on the literature found. The results will be presented based on four categories: (1) bibliometric variables of the included articles, (2) physical therapy interventions, (3) MV settings and (4) respiratory and metabolic variables used to monitor physical therapy interventions. A descriptive summary and synthesis of the study findings will be presented in results tables.

Potential discrepancies or uncertainties in the synthesis of information between the four reviewers will be discussed with a senior critical care physician (JGS).

\section{Stage 6: consultation}

If necessary, other experts on MV and physical therapy or the corresponding authors of selected articles will be consulted. A focused search of exercise physiology articles may be carried out if the findings extracted in the scoping review require physiological explanations not found in the original sources.

\section{Patient and public involvement}

The scoping review to follow on this protocol will not require the participation of any patients or the general public.

\section{ETHICS AND DISSEMINATION}

This scoping review does not require the ethics committee approval. This study seeks an integrated research approach including physiotherapists, a respiratory therapist, a critical care physician and a biomedical librarian. This will facilitate knowledge translation at the end of the study, integrating physical therapy and respiratory therapy in the ICU setting.

The knowledge translation of the scoping review results will be carried out based on the End-of-Grant objectives. ${ }^{36}$ These include three broad stages: diffusion (let it happen), dissemination (help to make it happen) and application (make it happen) ${ }^{36}$ Diffusion will take place in a peer-reviewed scientific journal covering as many users of intensive care knowledge as possible. Dissemination of the findings will take place through the presentation of the results and clinical recommendations in relevant international critical care congresses. Training to MV stakeholders in the use of the MV during physical therapy interventions will facilitate its application. The results of this scoping review could provide the basis for future studies on patient-ventilator interaction during physical therapy.

\section{Author affiliations}

${ }^{1}$ Servicio de Medicina Física y Rehabilitación, Facultad de Medicina, Clínica Alemana Universidad del Desarrollo, Santiago, Chile
${ }^{2}$ School of Physical Therapy, Facultad de Medicina, Clínica Alemana Universidad del Desarrollo, Santiago, Chile

${ }^{3}$ Bibliotecas Biomédicas, Facultad de Medicina, Clínica Alemana Universidad del

Desarrollo, Santiago, Chile

${ }^{4}$ Departamento de Paciente Crítico, Facultad de Medicina, Clínica Alemana Universidad del Desarrollo, Santiago, Chile

Contributors FG-S conceived the study. FG-S, AC-M, AJS and JGS contributed to the protocol design and plan. FG-S, AC-M and AJS developed and conducted the initial literature search. FG-S, AC-M, JMB, RPA and JGS worked collaboratively to several draft and revise the manuscript. All the authors made substantive intellectual contributions to the development of this protocol and approved the final version.

Funding The authors have not declared a specific grant for this research from any funding agency in the public, commercial or not-for-profit sectors.

Competing interests None declared.

Patient consent for publication Not required.

Provenance and peer review Not commissioned; externally peer reviewed.

Open access This is an open access article distributed in accordance with the Creative Commons Attribution Non Commercial (CC BY-NC 4.0) license, which permits others to distribute, remix, adapt, build upon this work non-commercially, and license their derivative works on different terms, provided the original work is properly cited, appropriate credit is given, any changes made indicated, and the use is non-commercial. See: http://creativecommons.org/licenses/by-nc/4.0/.

\section{REFERENCES}

1. Wunsch H, Linde-Zwirble WT, Angus DC, et al. The epidemiology of mechanical ventilation use in the United States. Crit Care Med 2010;38:1947-53.

2. Tobin MJ. Principles and practice of mechanical ventilation. 3rd edn. Mc. Graw-Hill, 2013.

3. Thille AW, Rodriguez P, Cabello B, et al. Patient-ventilator asynchrony during assisted mechanical ventilation. Intensive Care Med 2006;32:1515-22.

4. Moerer O. Effort-adapted modes of assisted breathing. Curr Opin Crit Care 2012;18:1-9.

5. Parry SM, Remedios L, Denehy L, et al. What factors affect implementation of early rehabilitation into intensive care unit practice? a qualitative study with clinicians. J Crit Care 2017;38:137-43.

6. Dubb R, Nydahl P, Hermes $C$, et al. Barriers and strategies for early mobilization of patients in intensive care units. Ann Am Thorac Soc 2016;13:724-30.

7. Devlin JW, Skrobik Y, Gélinas C, et al. Clinical practice guidelines for the prevention and management of pain, agitation/sedation, delirium, immobility, and sleep disruption in adult patients in the ICU. Crit Care Med 2018;46:e825-73.

8. Stockley RC, Hughes J, Morrison J, et al. An investigation of the use of passive movements in intensive care by UK physiotherapists. Physiotherapy 2010;96:228-33.

9. Schweickert WD, Pohlman MC, Pohlman AS, et al. Early physical and occupational therapy in mechanically ventilated, critically ill patients: a randomised controlled trial. Lancet 2009;373:1874-82.

10. Burtin C, Clerckx B, Robbeets C, et al. Early exercise in critically ill patients enhances short-term functional recovery. Crit Care Med 2009;37:2499-505.

11. Burke D, Gorman E, Stokes D, et al. An evaluation of neuromuscular electrical stimulation in critical care using the ICF framework: a systematic review and meta-analysis. Clin Respir J 2016;10:407-20.

12. Morris PE, Goad A, Thompson C, et al. Early intensive care unit mobility therapy in the treatment of acute respiratory failure. Crit Care Med 2008;36:2238-43.

13. Morris PE, Berry MJ, Files DC, et al. Standardized rehabilitation and hospital length of stay among patients with acute respiratory failure. JAMA 2016;315:2694-702.

14. Moss M, Nordon-Craft A, Malone D, et al. A randomized trial of an intensive physical therapy program for patients with acute respiratory failure. Am J Respir Crit Care Med 2016;193:1101-10.

15. Wright SE, Thomas K, Watson G, et al. Intensive versus standard physical rehabilitation therapy in the critically ill (EPICC): a multicentre, parallel-group, randomised controlled trial. Thorax 2018;73:213-21. 
16. Castro-Avila AC, Serón P, Fan E, et al. Effect of early rehabilitation during intensive care unit stay on functional status: systematic review and meta-analysis. PLoS One 2015;10:e0130722-21.

17. Schaller SJ, Anstey M, Blobner M, et al. Early, goal-directed mobilisation in the surgical intensive care unit: a randomised controlled trial. Lancet 2016;388:1377-88.

18. Stiller K. Physiotherapy in intensive care: an updated systematic review. Chest 2013;144:825-47.

19. Hodgson CL, Capell E, Tipping CJ. Early mobilization of patients in intensive care: organization, communication and safety factors that influence translation into clinical practice. Crit Care 2018;22.

20. Winkelman C, Sattar A, Momotaz H, et al. Dose of early therapeutic mobility: does frequency or intensity matter? Biol Res Nurs 2018;20:522-30.

21. Clarissa C, Salisbury L, Rodgers S, et al. Early mobilisation in mechanically ventilated patients: a systematic integrative review of definitions and activities. J Intensive Care 2019;7.

22. Akoumianaki E, Dousse N, Lyazidi A, et al. Can proportional ventilation modes facilitate exercise in critically ill patients? a physiological cross-over study. Ann Intensive Care 2017;7:64

23. Hodgson CL, Stiller K, Needham DM, et al. Expert consensus and recommendations on safety criteria for active mobilization of mechanically ventilated critically ill adults. Crit Care 2014;18.

24. Chen $\mathrm{Y}-\mathrm{H}$, Lin H-L, Hsiao H-F, et al. Effects of an additional pressure support level on exercise duration in patients on prolonged mechanical ventilation. J Formos Med Assoc 2015;114:1204-10.

25. Bailey P, Thomsen GE, Spuhler VJ, et al. Early activity is feasible and safe in respiratory failure patients. Crit Care Med 2007;35:139-45.

26. Hawkins P, Johnson LC, Nikoletou D, et al. Proportional assist ventilation as an aid to exercise training in severe chronic obstructive pulmonary disease. Thorax 2002:57:853-9.
27. Peters MDJ, Godfrey $\mathrm{CM}$, Khalil $\mathrm{H}$, et al. Guidance for conducting systematic scoping reviews. Int J Evid Based Healthc 2015;13:141-6.

28. Munn Z, Peters MDJ, Stern C, et al. Systematic review or scoping review? guidance for authors when choosing between a systematic or scoping review approach. BMC Med Res Methodol 2018;18:143.

29. Khalil H, Peters M, Godfrey CM, et al. An evidence-based approach to scoping reviews. Worldviews Evid Based Nurs 2016;13:118-23.

30. Arksey H, O'Malley L. Scoping studies: towards a methodological framework. Int J Soc Res Methodol 2005;8:19-32.

31. Tricco AC, Lillie E, Zarin W, et al. PRISMA extension for scoping reviews (PRISMA-ScR): checklist and explanation. Ann Intern Med 2018;169:467.

32. HigginsJPT, Green S, eds. Cochrane handbook for systematic reviews of interventions version 5.1.0 [updated March 2011]. The Cochrane Collaboration, 2011.

33. Liberati A, Altman DG, Tetzlaff J, et al. The PRISMA statement for reporting systematic reviews and meta-analyses of studies that evaluate health care interventions: explanation and elaboration. $J$ Clin Epidemiol 2009;62:e1-34.

34. Jolley SE, Moss M, Needham DM, et al. Point prevalence study of mobilization practices for acute respiratory failure patients in the United States. Crit Care Med 2017;45:205-15.

35. Reid JC, Unger J, McCaskell D, et al. Physical rehabilitation interventions in the intensive care unit: a scoping review of 117 studies. J Intensive Care 2018;6.

36. Canadian Institutes of Health Research. Guide to knowledge translation planning at CIHR: integrated and End-of-grant approaches. Canadian Institutes of Health Research, 2012. www. cihr-irsc.gc.ca/e/45321.htm 